\title{
ON GRONWALL AND WENDROFF TYPE INEQUALITIES
}

\section{J. ABRAMOWICH}

\begin{abstract}
It is shown how Gronwall's Lemma and the extension to many variables given by $\mathrm{W}$. Walter may be derived using the simple method of recursion. This same method is used to extend this result and to derive a more general Wendroff type inequality.

Upper and lower bounds for the Neumann series in the case of two independent variables are given.
\end{abstract}

Introduction. W. Walter [1] extended Gronwall's Lemma to more than one independent variable using monotone operators; D. R. Snow used Riemann's method of integration for the case of two independent variables. In this paper we give a very simple proof of these results using the method of recursion. Using this method we extend the result of $W$. Walter and a result of Bondge, Pachpatte and Walter on Wendroff type inequalities.

1. The method. Let $a, b, s, t$ and $x$ denote real $n$-vectors and $u(x)$ a scalar function. If $a=\left(a_{1}, \ldots, a_{n}\right), x=\left(x_{1}, \ldots, x_{n}\right), t=\left(t_{1}, \ldots, t_{n}\right)$ and $b=\left(b_{1}, \ldots, b_{n}\right)$ we shall denote the integral

$$
\int_{a_{1}}^{x_{1}} \cdots \int_{a_{n}}^{x_{n}} u\left(t_{1}, \ldots, t_{n}\right) d t_{1} \cdots d t_{n}
$$

by $J_{a x} u(t)$ and the region $\left[0, b_{1}\right] \times \cdots \times\left[0, b_{n}\right]$ in $E_{n}$ by $D(b)$.

If $T(x, u(t))$ is a real functional, we shall say that the inequality $u(x) \leqslant T(x, u(t))$ is recursive if the r.h.s. can be substituted for $u(t)$ on the r.h.s. and the process repeated indefinitely with preservation of the inequality.

Let $f(x), u(x)$ and $v(x) \geqslant 0$ be bounded integrable functions in $D(b)$. Under these conditions the inequality

$$
u(x) \leqslant f(x)+J_{0 . x}(v(t) u(t))
$$

is recursive in $D(b)$.

Substituting the right-hand side of $(1.1)$ for $u(t)$ under the integral sign and interchanging the order of integration we obtain

$$
u(x) \leqslant f(x)+J_{0 x}(v(t) f(t))+J_{0 x}\left(v(t) g_{1}(x, t) u(t)\right)
$$

Received by the editors January 18, 1982.

1980 Mathematics Subject Classification. Primary 35C05.

Key nords and phrases. Integral inequality, (ironwall, Wendroff, recursion. 
where $g_{1}(x, t)=J_{t x}(v(s))$. Repeating the procedure (assuming we may interchange the order of integration) we obtain

$$
u(x) \leqslant f(x)+J_{0 x}\left(v(t) f(t) \sum_{i=0}^{m} g_{i}(x, t)\right)+J_{0 x}\left(u(t) v(t) g_{m+1}(x, t)\right)
$$

where

$$
g_{0}(x, t)=1, \quad g_{k+1}(x, t)=J_{t x}\left(v(s) g_{k}(x, s)\right), \quad k \geqslant 0 .
$$

It is easily seen that

$$
\left|J_{0 x}\left(u(t) v(t) g_{m+1}(x, t)\right)\right| \leqslant \frac{M}{((m+1) !)^{n}}
$$

for $x \in D(b)$ where $M$ is independent of $x$ and letting $m \rightarrow \infty$ in (1.2) we obtain

$$
u(x) \leqslant f(x)+J_{0 x}\left(f(t) v(t) N_{v}(x, t)\right), \quad x \in D(b),
$$

where $N_{v}(x, t)=\sum_{i=0}^{\infty} g_{i}(x, t)$ is the so-called Neumann series for the function $v(x)$. This is W. Walter's solution of (1.1). The special case of $f(x)=C=$ constant may be solved in the same way (or with greater effort obtained from (1.3)) to give

$$
u(x) \leqslant C \sum_{k=0}^{\infty} I_{k}(x)=C \underline{N}_{,}(x), \quad x \in D(b),
$$

where

$$
I_{0}(x)=1, \quad I_{k+1}(x)=J_{0 x}\left(v(t) I_{k}(t)\right), \quad k \geqslant 0 .
$$

2. A Gronwall type inequality. Let $f(x)$ and $u(x)$ be as in (1.1) and $v(x, t) \geqslant 0$ be a bounded, integrable function for $x \in D(b), t \in D(x)$. Then the inequality

$$
u(x) \leqslant f(x)+J_{0 x}(v(x, t) u(t))
$$

is recursive. This inequality may be solved in exactly the same way as (1.1) and we shall omit the details. The solution is

$$
u(x) \leqslant f(x)+J_{0 x}\left(f(t) M_{v}(x, t)\right), \quad x \in D(b),
$$

where $M_{v}(x, t)=\sum_{n=0}^{\infty} v_{n}(x, t)$, and

$$
v_{0}(x, t)=v(x, t), \quad v_{k+1}(x, t)=J_{t x}\left(v(s, t) v_{k}(x, s)\right), \quad k \geqslant 0 .
$$

In the particular case that $v(x, t)=p(x) w(t),(2.2)$ reduces to

$$
u(x) \leqslant f(x)+p(x) J_{0 x}\left(f(t) N_{p w}(x, t)\right)
$$

where $N_{p w}(x, t)$ is the Neumann series for the function $p(x) w(x)$.

As an application, consider the inequality

$$
u(x) \leqslant f(x)+J_{0 x}(p(t) u(t))+J_{0 x}\left(q(t) J_{0 t}(r(s) u(s))\right)
$$

where $p(t) \geqslant 0, q(t) \geqslant 0, r(t) \geqslant 0$ and $u(t)$ are bounded integrable functions in $D(b)$. Interchanging the order of integration in the last term (assuming it is valid) and setting $Q(x, t)=J_{t x}(q(t))$ we obtain

$$
u(x) \leqslant f(x)+J_{0 x}(v(x, t) u(t))
$$

where $v(x, t)=p(t)+Q(x, t) r(t)$. 
3. Wendroff type inequalities. Let $f(x), v(x)$ and $u(x)$ be as in (1.1) and $M(x)=\sup _{t \in D(x)} f(t)$. Then the inequality

$$
u(x) \leqslant f(x)+J_{0 x}(v(t) u(t))
$$

has the solution

$$
u(x) \leqslant M(x) \underline{N}_{v}(x)
$$

where $\underline{N}_{v}(x)$ is as in (1.4). The proof is simple. From (3.1) we have

$$
\begin{aligned}
u(x) & \leqslant M(x)+J_{0 x}(v(t) u(t)) \\
& \leqslant M(x)+J_{0 x}\left(v(t)\left(M(t)+J_{0 t}(v(s) u(s))\right)\right) \\
& \leqslant M(x)+M(x) J_{0 x}(v(t))+J_{0 x}\left(v(t) J_{0 t}(v(s) u(s))\right)
\end{aligned}
$$

and the proof is much the same as for (1.4). The inequality $\underline{N}_{v}(x) \leqslant \exp \left(J_{0 x}(v(t))\right)$ $(v(t) \geqslant 0)$ is easily established so that in the case $M(x) \geqslant 0(3.2)$ may be written

$$
u(x) \leqslant M(x) \exp \left(J_{0 x}(v(t))\right)
$$

which, however, is a much 'coarser' inequality than (3.2). This generalizes a result due to Bondge, Pachpatte and Walter [2]. Note that (3.3) need not be valid if $f(x)<0$ as the example

$$
u(x, y)=-1+\int_{0}^{x} \int_{0}^{y} u(s, t) d s d t \quad(x, y \text { scalars })
$$

with the solution $u(x, y)=-\sum_{k=0}^{\infty}(x y)^{k} /(k !)^{2}$ shows, contrary to the assertion in [2].

A result similar to (3.2) may be established for the inequality (2.1) in exactly the same way:

$$
u(x) \leqslant M(x)\left[1+J_{0 x}\left(M_{v}(x, t)\right)\right] .
$$

As an application of this result, (1.1) may be transformed into the inequality

$$
u(x) \leqslant f(x)+J_{0 x}(v(t) f(t))+J_{0 x}(p(x, t) u(t))
$$

where $p(x, t)=v(t) J_{t x}(v(s))$. From (3.4) we get

$$
u(x) \leqslant M(x)\left[1+J_{0 x}\left(M_{p}(x, t)\right)\right]
$$

where

$$
M(x)=\sup _{s \in D(x)}\left[f(s)+J_{0 s}(v(t) f(t))\right] .
$$

4. Some bounds in the case of two variables. The solutions of the inequalities in the previous sections are in terms of repeated integrals which may be inconvenient to use. If in (1.1) $v(t)=v_{1}\left(t_{1}\right) \cdots v_{n}\left(t_{n}\right)$, the function $N_{v}(x, t)$ in (1.3) simplifies to

$$
N_{v}(x, t)=E_{n}\left(J_{t x}(v(t))\right)
$$

where

$$
E_{n}(z)=\sum_{k=0}^{\infty} \frac{z^{k}}{(k !)^{n}}
$$


Though (4.1) holds only in the case when $v(t)$ is a product of functions of one variable, it is possible to obtain lower and upper bounds for $N_{v}(x, t)$ of similar form. This is difficult to do for any number of independent variables and we shall consider the case of two independent variables only.

For the remainder of this section, we shall abandon vector notation so that $x, y$, etc., shall denote scalar variables. Partial derivatives shall be denoted by subscripts or by $D_{x}, D_{y}, D_{x y}$, etc. Throughout this section $J_{s t}^{x y}(v)$ shall denote the integral

$$
\int_{s}^{x} \int_{t}^{y} v(p, q) d p d q
$$

and in the case that both lower limits of integration are zero, by $J_{x v}(v)$. The variables of integration shall be shown explicitly only if necessary for clarity. By $R(a, b)$ we shall denote the rectangle $[0, a] \times[0, b]$ in the Euclidean space $E_{2}$.

LEMMA 1. Let $I_{n}(x, y ; s, t)$ denote the integrals

$$
\begin{aligned}
I_{0}(x, y ; s, t) & =1, \\
I_{n+1}(x, y ; s, t) & =J_{s t}^{x y}\left(v(p, q) I_{n}(x, y ; p, q)\right), \quad n \geqslant 0,
\end{aligned}
$$

and

$$
K_{n}(x, y ; s, t)=\frac{1}{(n !)^{2}}\left[J_{s t}^{x y}(v)\right]^{n}, \quad n \geqslant 0 .
$$

Let

$$
Q(x, y ; s, t)=\int_{s}^{x} v(p, y) d p \int_{t}^{y} v(x, q) d q / v(x, y) J_{s t}^{x y}(v),
$$

where $0 \leqslant s \leqslant x$ and $0 \leqslant t \leqslant y$. If

$$
\begin{aligned}
& r=\min \left(1, \inf _{(x, y) \in R(a, b)} Q(x, y ; s, t)\right) \text { and } \\
& R=\max \left(1, \sup _{(x, y) \in R(a, b)} Q(x, y ; s, t)\right)
\end{aligned}
$$

and if $v(x, y) \geqslant 0,(x, y) \in R(a, b)$, then

$$
R^{-n} K_{n}(x, y ; s, t) \leqslant I_{n}(x, y ; s, t) \leqslant r^{-n} K_{n}(x, y ; s, t), \quad n \geqslant 0 ;
$$

furthermore, $r=R=1$ iff $v(x, y)=v_{1}(x) v_{2}(y)$ in which case equality holds in (4.4).

Proof. For $n=0,(4.4)$ is trivial and by the induction hypothesis

$$
I_{n+1}(x, y ; s, t)=J_{s t}^{x y}\left(v I_{n}\right) \leqslant r^{-n} J_{s t}^{x y}\left(v K_{n}\right) \leqslant R^{-n} J_{s t}^{x y}\left(v K_{n}\right)
$$

and we need only establish

$$
R^{-1} K_{n+1}(x, y ; s, t) \leqslant J_{s t}^{x y}\left(v K_{n}\right) \leqslant r^{-1} K_{n+1}(x, y ; s, t) .
$$

A straightforward calculation gives

$$
D_{x y} K_{n+1}(x, y ; s, t)=K_{n}(x, y ; s, t) v(x, y)\left[\frac{n Q(x, y ; s, t)+1}{(n+1)}\right]
$$


which gives

$$
\begin{aligned}
r K_{n}(x, y ; s, t) v(x, y) & \leqslant D_{x y} K_{n+1}(x, y ; s, t) \\
& \leqslant R K_{n}(x, y ; s, t) v(x, y) .
\end{aligned}
$$

Integrating this inequality and using the fact that $K_{n+1}(s, y ; s, t)=K_{n+1}(x, t ; s, t)$ $=K_{n+1}(s, t ; s, t)=0$ we obtain the desired result.

To prove the second assertion we note that $r=R=1$ iff $Q(x, y ; s, t)=1$. If $v(x, y)=v_{1}(x) v_{2}(y)$ then obviously this condition is satisfied. Suppose now that $Q(x, y ; s, t)=1$. This implies that

$$
D_{x} V D_{y} V-V D_{x y} V=0, \quad V=J_{s i}^{x y}(v) .
$$

Since $V>0$ for $x>0, y>0$ setting $U=\ln (V)$ we get $D_{x y} U=0$ from which we get $V=V_{1}(x) V_{2}(y)$ and $v(x, y)=D_{x y} V=v_{1}(x) v_{2}(y)$. This completes the proof of the lemma.

THEOREM 1. Let

$$
N_{v}(x, y ; s, t)=\sum_{k=0}^{\infty} I_{k}(x, y ; s, t)
$$

denote the Neumann series for the function $v(x, y) \geqslant 0,0 \leqslant s \leqslant x \leqslant a, 0 \leqslant t \leqslant y \leqslant b$, and let $r, R$ be the numbers defined in (4.3). Then

$$
E_{2}\left(R^{-1} J_{s t}^{x y}(v)\right) \leqslant N_{v}(x, y ; s, t) \leqslant E_{2}\left(r^{-1} J_{s t}^{x y}(v)\right)
$$

for $0 \leqslant s \leqslant x, 0 \leqslant t \leqslant y$ where $E_{2}(z)$ is as in (4.2).

The proof is an immediate consequence of Lemma 1 .

As an example, if $v(x, y)=x+y$ then $r=1, R=9 / 8$ and

$$
E_{2}\left(8 / 9 J_{s t}^{x y}(v)\right) \leqslant N_{v}(x, y ; s, t) \leqslant E_{2}\left(J_{s t}^{x y}(v)\right)
$$

for $x \geqslant 0, y \geqslant 0$.

These inequalities are particularly useful if $Q(x, y ; s, t)$ has a local maximum and a local minimum in the quadrant $x, y \geqslant 0$.

Using (4.5) we can put (3.2) in the simpler form

$$
u(x, y) \leqslant M(x, y) E_{2}\left(r^{-1} J_{x y}(v)\right)
$$

provided $M(x, y) \geqslant 0$.

5. Some extensions of results. It is evident that the method of recursion is applicable to systems of inequalities of the form (1.1) of (2.1) and to more general functional inequalities. We shall not, however, consider these generalizations here.

An example of the flexibility of the method of recursion is the 'partial integration' of inequalities. Suppose we have the inequality

$$
u(x) \leqslant f(x, u(x))+J_{0 x}(v(t) u(t))
$$

and that $v(t) \geqslant 0$. Then we may consider the first term on the r.h.s. as a known function of $x$ and using (1.3) we obtain

$$
u(x) \leqslant f(x, u(x))+J_{0 x}\left(v(t) f(t, u(t)) N_{v}(x, t)\right)
$$


which may be more useful than the original. If the inequality (5.1) is the result of integrating the differential inequality

$$
u(x)_{x_{1} x_{2} \cdots x_{n}} \leqslant g(x, u(x))+v(x) u(x),
$$

where the subscripts on the 1.h.s. denote partial differentiation, then (5.2) can be considered as a partial integration of this differential inequality.

As an example of the application of (5.2), consider the inequality (1.1) in the case when the condition $v(x) \geqslant 0$ is not satisfied. Writing $v(x)=p(x)-q(x), p(x) \geqslant 0$, $q(x) \geqslant 0$, and using (5.2), we obtain

$$
\begin{aligned}
u(x) \leqslant & f(x)+J_{0 x}\left(p(t) f(t) N_{p}(x, t)\right)-J_{0 x}\left(J_{0 t}(q(s) u(s)) N_{p}(x, t)\right) \\
& -J_{0 x}(q(t) u(t)) .
\end{aligned}
$$

If a lower bound for $u(x)$ is known, say $u(x) \geqslant C$, then a useful upper bound may be obtained by replacing $u(t)$ on the r.h.s. by $C$.

\section{REFERENCES}

1. W. Walter, Differential and integral inequalities, Springer-Verlag, New York, 1970.

2. B. K. Bondge, B. G. Pachpatte and W. Walter, On generalized Wendroff type inequalities and their applications, Nonlinear Analysis, Theory, Methods \& Applications 4 (1980).

3. D. R. Snow, $A$ two independent variable Gronwall type inequality, Inequalities III, Academic Press, New York, 1971, pp. 330-340.

6 Highview Drive, Doncaster, Victoria 3108, Australia 